\title{
ON MANIFOLD SPINES AND CYCLIC PRESENTATIONS OF GROUPS
}

\author{
ALBERTO CAVICCHIOLI \\ Dipartimento di Matematica, Università di Modena \\ Via Campi 213/B, 41100 Modena, Italy \\ E-mail: cavicchioli@dipmat.unimo.it \\ FRIEDRICH HEGENBARTH \\ Dipartimento di Matematica, Università di Milano \\ Via C. Saldini 50, 20133 Milano, Italy \\ E-mail:dipmat@imiucca.csi.unimi.it \\ DUŠAN REPOV ̌ \\ Institute of Mathematics, University of Ljubljana \\ P.O. Box 2964, Ljubljana 1001, Slovenia \\ E-mail: dusan.repovs@fmf.uni-lj.si
}

\begin{abstract}
This is a survey of results and open problems on compact 3-manifolds which admit spines corresponding to cyclic presentations of groups. We also discuss questions concerning spines of knot manifolds and regular neighborhoods of homotopically PL embedded compacta in 3-manifolds.
\end{abstract}

1. Spines of 3-manifolds. Let

$$
G=\left\langle x_{1}, x_{2}, \ldots, x_{n}: g_{1}, g_{2}, \ldots, g_{m}\right\rangle
$$

be a finite group presentation with $n$ generators and $m$ relators, $n \geq m$. We can associate to $G$ a canonical 2-complex $K_{G}$, with one vertex $v$, such that $\Pi_{1}\left(K_{G}\right)$ is presented by $G$. Its 1-skeleton $K_{G}^{(1)}$ is a bouquet of $n$ circles with a fixed orientation, also denoted

1991 Mathematics Subject Classification: Primary 57M05; Secondary 57M12.

Work performed under the auspices of the G.N.S.A.G.A. of the C.N.R. (National Research Council) of Italy and partially supported by the Ministero per la Ricerca Scientifica e Tecnologica of Italy within the projects Geometria Reale e Complessa and Topologia and by the Ministry of science and Technology of the Republic of Slovenia grant No. J1-7039-0101-95.

The paper is in final form and no version of it will be published elsewhere. 
by $x_{1}, x_{2}, \ldots, x_{n}$. The 2-cells $c_{j}$ of $K_{G}$ correspond bijectively to the relators $g_{j}$ which determine closed curves as the corresponding attaching maps $h_{j}: \partial B^{2} \rightarrow K_{G}^{(1)}$.

Given such a 2-complex $K_{G}$, the following questions arise:

Problem 1. Does $K_{G}$ embed into the interior of some compact connected orientable 3 -manifold $M$ as its spine, i.e. either $M$ (if $\partial M \neq \emptyset$ ) or $M \backslash$ (open 3-cell) (if $\partial M=\emptyset$ ) collapses onto $K_{G}$ ?

Problem 2. If so, classify the topological structure of all 3-manifolds which admit $K_{G}$ as spine.

Problem 3. Construct examples of nonhomeomorphic compact 3-manifolds $M_{i}, i=$ 1,2 , in the 3 -sphere with connected boundary $\partial M_{1} \cong \partial M_{2}$ of arbitrary genus such that $M_{1}$ and $M_{2}$ have the same spine $K_{G}$, where $G$ is not a nontrivial free product.

Suppose that $K_{G}$ embeds into a compact 3-manifold $M$ as its spine. A regular neighborhood of $K_{G}^{(1)}$ in $M$ is a handlebody $H$ of genus $n$, each 1-handle corresponding to a generator $x_{i}$ of $G$. The boundary $\partial H$ intersects $K_{G}$ in a collection of pairwise disjoint simple closed curves $\alpha_{j}$ which run around the handles according to the relators $g_{j}$ of $G$. The curves $\alpha_{j}$ are the boundaries of the 2-discs in the closure of the complement in $K_{G}$ of a regular neighborhood of its 1-skeleton. These discs thicken to cylinders $B_{j}^{2} \times I$, $I=[0,1]$, where $\partial B_{j}^{2} \times I$ must be identified with a regular neighborhood of $\alpha_{j}$ in $\partial H$. The resulting manifold is a regular neighborhood of $K_{G}$ in $M$ and hence it is homeomorphic to $M$.

The $(m+1)$-tuple $\left(H ; \alpha_{1}, \alpha_{2}, \ldots, \alpha_{m}\right)$ is the Heegaard diagram of $M$.

The following represents a first criterion to answer Problem 1 (see [11]).

THEOREM $1 . K_{G}$ is a spine of a compact connected (orientable) 3-manifold if and only if it is possible to draw pairwise disjoint simple closed curves $\alpha_{j}$ on the boundary of an (orientable) handlebody $H$ with $n$ handles (one handle for each $x_{i}$ ) such that $\alpha_{j}$ reads the relator $g_{j}$ on $H$.

Now cut each handle of $H$ along a thickened disc. The curves $\alpha_{j}$ give rise to $\operatorname{arcs} \gamma_{\ell}$ running on a 2 -sphere with $2 n$ holes, $D_{i}$ and $\bar{D}_{i}$ say. Let $e_{i}^{k}$ and $\bar{e}_{i}^{k}, k=1,2, \ldots, r(i)$, denote the intersection points of the $\operatorname{arcs} \gamma_{\ell}$ with $\partial D_{i}$ and $\partial \bar{D}_{i}$, respectively. For each $i$, they are assumed to be ordered clockwise (resp. counterclockwise) according to an orientation of the 2-sphere. Obviously, identifying $D_{i}$ with $\bar{D}_{i}$, such that $e_{i}^{k}$ falls onto $\bar{e}_{i}^{k}$, yields the initial Heegaard diagram of $M$.

Let $E$ denote the set consisting of all points $e_{i}^{k}$ and $\bar{e}_{i}^{k}, k=1,2, \ldots, r(i)$, and define three permutations $A, B$ and $C$ on $E$, as follows:

(1) $A$ is the product of the disjoint transpositions interchanging the endpoints of the $\operatorname{arcs} \gamma_{\ell}$;

(2) $B$ is the involutory permutation sending $e_{i}^{k}$ onto $\bar{e}_{i}^{k}$;

(3) $C=\prod_{i=1}^{n}\left(e_{i}^{1} \cdots e_{i}^{r(i)}\right)\left(\bar{e}_{i}^{r(i)} \cdots \bar{e}_{i}^{1}\right)$. 
The following gives a second criterion to answer Problem 1. It was first proved in [19] for closed 3-manifolds and then extended to the boundary case in [2].

THEOREM 2. Let $G$ be a finite group presentation with $n$ generators and $m$ relators, $n \geq m$. Then $K_{G}$ is a spine of a connected compact orientable 3 -manifold $M$ with nonvoid boundary if and only if $|A|-|C|+2=|A C|$.

The number of components of $\partial M$ equals $|A C, B C|$. If $\partial M$ is connected, then it is the closed orientable surface of genus $n-m$.

Here $\left|p_{1}, \ldots, p_{t}\right|$ denotes the number of orbits of the group generated by the permutations $p_{1}, \ldots, p_{t}$.

The permutations $A$ and $B$ are uniquely determined by the group presentation $G$. A search (possibly by a computer program) of all permutations $C$ satisfying the formulas of Theorem 2 yields all bordered compact orientable 3-manifolds (in general, with repetitions) having the same spine $K_{G}$.

2. Cyclic presentations. Let $F_{n}$ be the free group on $n$ generators $x_{1}, x_{2}, \ldots, x_{n}$. Let $\theta: F_{n} \rightarrow F_{n}$ denote the automorphism such that $\theta\left(x_{i}\right)=x_{i+1}, i \bmod n$. For any word $w \in F_{n}$, define

$$
G_{n}(w)=\frac{F_{n}}{R},
$$

where $R$ is the normal closure in $F_{n}$ of the set

$$
\left\{w, \theta(w), \ldots, \theta^{n-1}(w)\right\} .
$$

A group $G$ is said to have a cyclic presentation if $G$ is isomorphic to $G_{n}(w)$ for some $n$ and $w$ (see for example [14]).

Problem 4. What cyclic presentations of groups correspond to spines of closed orientable 3-manifolds?

It seems very plausible that the resulting 3-manifolds appear to be cyclic coverings of the 3-sphere branched over appropriate knots or links.

Problem 5. What classes of knots or links arise from branched coverings encoded by cyclic presentations?

We are going to discuss two interesting cases of cyclic presentations yielding 3manifolds and then classify the topological structure of these manifolds.

The Fibonacci groups are abstract groups defined by the presentations

$$
\mathcal{F}(r, m)=\left\langle x_{1}, x_{2}, \ldots, x_{m}: x_{i} x_{i+1} \cdots x_{i+r-1} x_{i+r}^{-1}=1(\text { indices } \bmod m)\right\rangle
$$

for any two positive integers $r$ and $m(\geq 2)$. These groups were investigated by a number of mathematicians (see for example [3], [8], [9], [10], [13], [14], [15] and [16]). It was shown that the groups $\mathcal{F}(2,2 n), n \geq 2$, are interesting from geometrical point of view (see the quoted papers).

TheOREM 3. Let $K(2,2 n)$ be the canonical 2-complex associated to the Fibonacci group $\mathcal{F}(2,2 n), n \geq 2$. Then the unique closed orientable prime 3 -manifold $M(2,2 n)$ with 
spine $K(2,2 n)$ is the $n$-fold cyclic covering of the 3 -sphere $\mathbb{S}^{3}$ branched over the figureeight knot. In particular, $M(2,4)$ is the lens space $L(5,2)$. Further, $M(2,2 n), n \geq 3$, has Heegaard genus two since it is a double cyclic covering of $\mathbb{S}^{3}$ branched over the closed 3 -strings braid $\left(\sigma_{1} \sigma_{2}^{-1}\right)^{n}$, where $\sigma_{1}$ and $\sigma_{2}$ are canonical generators.

As proved in [9], $M(2,2 n)$ is hyperbolic for $n \geq 4$ and Euclidean for $n=3$. Thus we have the following corollaries (see [21] for a survey on the geometries of 3 -manifolds).

COROLlary 4. The Fibonacci group $\mathcal{F}(2,2 n)$ is isomorphic to a properly discontinuous cocompact group of isometries which acts without fixed points on a space $X_{n}$, where $X_{2}=\mathbb{S}^{3}$ (spherical), $X_{3}=\mathbb{E}^{3}$ (Euclidean), and $X_{n}=\mathbb{H}^{3}$ (hyperbolic) for $n \geq 4$.

COROLlaRY 5. Let $\mathcal{O}(n)$ be the orbifold with underlying space $\mathbb{S}^{3}$ and the singular set the figure-eight knot with branched index $n$. Then $\mathcal{O}(n)$ is spherical for $n<3$, Euclidean for $n=3$, and hyperbolic for $n>3$.

It was further proved that $\mathcal{F}(r, 2), r \geq 2$, and $\mathcal{F}(n-1, n), n \geq 3$, correspond to spines of Seifert fibered 3-manifolds (see [3] and [8]). However, we have verified by a direct computation (use the computer program mentioned at the end of Section 1) that the Fibonacci groups $\mathcal{F}(2,5), \mathcal{F}(2,7), \mathcal{F}(3,5), \mathcal{F}(3,6), \mathcal{F}(3,7), \mathcal{F}(3,8), \mathcal{F}(4,3), \mathcal{F}(4,6)$, and $\mathcal{F}(5,4)$ do not correspond to spines of closed 3 -manifolds. It seems natural for example to think that there are no 3 -manifolds with spines corresponding to the Fibonacci groups $\mathcal{F}(2,2 n+1), n \geq 2$.

The Sieradski groups are abstract groups defined by presentations

$$
\mathcal{S}(r, n)=\left\langle x_{1}, x_{2}, \ldots, x_{n}: x_{i} x_{i+2} \cdots x_{i+2 r-2}=x_{i+1} \cdots x_{i+2 r-3}(\text { indices } \bmod n)\right\rangle
$$

for any two positive integers $r$ and $n(\geq 2)$. For example, $n=2$ gives the presentation $\left\langle x_{1}, x_{2}: x_{1}^{r}=x_{2}^{r-1} \wedge x_{2}^{r}=x_{1}^{r-1}\right\rangle$, and the corresponding canonical 2-complex can be checked to be a spine of the lens space $L(2 r-1,1)$. These groups, introduced in [22] for $r=2$, were successively obtained in [12], [13], and [23] from different geometric constructions. The following will appear in [5].

TheOREM 6. The Sieradski groups $\mathcal{S}(r, n)$ correspond to a spine of a closed connected orientable 3-manifold $\mathcal{M}(r, n)$. Moreover, $\mathcal{M}(r, n)$ is homeomorphic to the $n$-fold cyclic covering of the 3 -sphere $\mathbb{S}^{3}$ branched over the torus knot of type $(2 r-1,2)$, i.e. $\mathcal{M}(r, n)$ is the Brieskorn manifold $M(2,2 r-1, n)$ in the sense of [17]. In particular, $\mathcal{M}(r, 2)$ is the lens space $L(2 r-1,1)$. Finally, $\mathcal{M}(r, n)$ admits the structure of a Seifert bundle over the 2 -orbifold $\mathbb{S}^{2}(2,2 r-1, n)$ which is a 2 -sphere $\mathbb{S}^{2}$ with three cone points with cone angles $\pi, \frac{2 \pi}{2 r-1}$, and $\frac{2 \pi}{n}$.

Note that this theorem, for $r=2$, answers in the affirmative an open problem suggested by the referee in [22].

In particular, we have the following corollaries.

COROLlaRY 7. The Sieradski group $\mathcal{S}(2, n)$ is isomorphic to a properly discontinuous cocompact group of isometries which acts without fixed points on a space $X_{n}$, where $X_{n}=\mathbb{S}^{3}$ for $n<6, X_{6}=\mathrm{Nil}$, and $X_{n}=\widetilde{\mathrm{SL}}(2 ; \mathbb{R})$ for $n>6$. 
COROLLARY 8. Let $\Theta(n)$ be the orbifold with underlying space $\mathbb{S}^{3}$ and the singular set the trefoil knot with branched index $n$. Then $\Theta(n)$ is spherical for $n<6$, a Nil-orbifold for $n=6$, and $a \widetilde{\mathrm{SL}}(2 ; \mathbb{R})$-orbifold for $n>6$.

We conclude the section with an open problem on a class of cyclic presentations $G(h, k, n)$ which includes the Fibonacci groups $\mathcal{F}(2, n)$ for $h=1 \wedge k=2$, and the Sieradski groups $\mathcal{S}(2, n)$ for $h=2 \wedge k=1$.

Problem 6. Determine all the pairs $(h, k)$ of positive integers for which the cyclic presentations

$$
G(h, k, n)=\left\langle x_{1}, x_{2}, \ldots, x_{n}: x_{i} x_{i+h} x_{i+k}^{-1}=1(\text { indices } \bmod n)\right\rangle
$$

correspond to spines of closed 3-manifolds $M(h, k, n)$ for infinitely many $n$. Then classify the topological structure of these classes of manifolds.

3. Equivalent spines. In this section we turn our attention to Problem 3. Let $K$ be an oriented tame (smooth or PL) knot in the right-hand oriented 3-sphere $\mathbb{S}^{3}$. Take a tubular neighborhood $V$ of $K$ in $\mathbb{S}^{3}$ and let $M=\mathbb{S}^{3} \backslash \dot{V}$ be the knot manifold of $K$.

In [4] the following result on spines of knot manifolds was proved.

Theorem 9. Suppose that $\eta$ is a Wirtinger presentation (with deficiency one) of the knot group of $K$. Then the canonical 2-complex $K_{\eta}$ is a spine of the knot manifold of $K$.

Theorem 9 directly implies the existence of many examples of nonhomeomorphic compact 3-manifolds $M_{1}, M_{2} \subset \mathbb{S}^{3}$ with $\partial M_{i} \cong \mathbb{S}^{1} \times \mathbb{S}^{1}$ which admit the same spine. The following extends earlier constructions on connected sums of torus knots, given in [18].

TheOREM 10. Let $K_{1} \subset \mathbb{S}^{3}$ be an invertible nonamphicheiral knot and let $K_{2} \subset \mathbb{S}^{3}$ be an arbitrary knot. Then the knot manifolds of $K_{1} \# K_{2}$ and $\bar{K}_{1} \# K_{2}\left(\bar{K}_{1}\right.$ being the mirror-image of $K_{1}$ ) have a common spine. If $K_{2}$ is also nonamphicheiral, then these knot manifolds are not homeomorphic.

In particular, for any invertible nonamphicheiral knot $K$, the knot manifolds of $K \# K$ and $\bar{K} \# K$ are not homeomorphic but they do possess a common spine.

To construct examples with boundary genus greater than one, we need the concept of $\theta$-manifold, introduced in [6]. Let $\theta\left(K_{1}, K_{2}, K_{3}\right)$ be the oriented $\theta$-curve, embedded in $\mathbb{S}^{3}$, and formed by two points joined with three arcs knotted according to the oriented knots $K_{1}, K_{2}$, and $K_{3}$, respectively. Let $M\left(K_{1}, K_{2}, K_{3}\right)$ be the closure of the complement of a regular neighborhood of this graph in $\mathbb{S}^{3}$. Then $M\left(K_{1}, K_{2}, K_{3}\right)$ is an irreducible 3manifold, with boundary of genus two, called a $\theta$-manifold. Obviously, this construction can be generalized to give manifolds with higher boundary genus.

The Torus Decomposition Theorem for a compact irreducible 3-manifold $M$ asserts that in $M$ there exists a collection of incompressible tori that separate $M$ into atoroidal or Seifert fibered components. These decomposing tori for the $\theta$-manifold $M\left(K_{1}, K_{2}, K_{3}\right)$ are three annuli, $T_{1}, T_{2}$ and $T_{3}$ say, which run around the knotted parts of the $\theta$-curve $\theta\left(K_{1}, K_{2}, K_{3}\right)$ as shown in Figure 1.

In $[6]$ the following result was proved. 


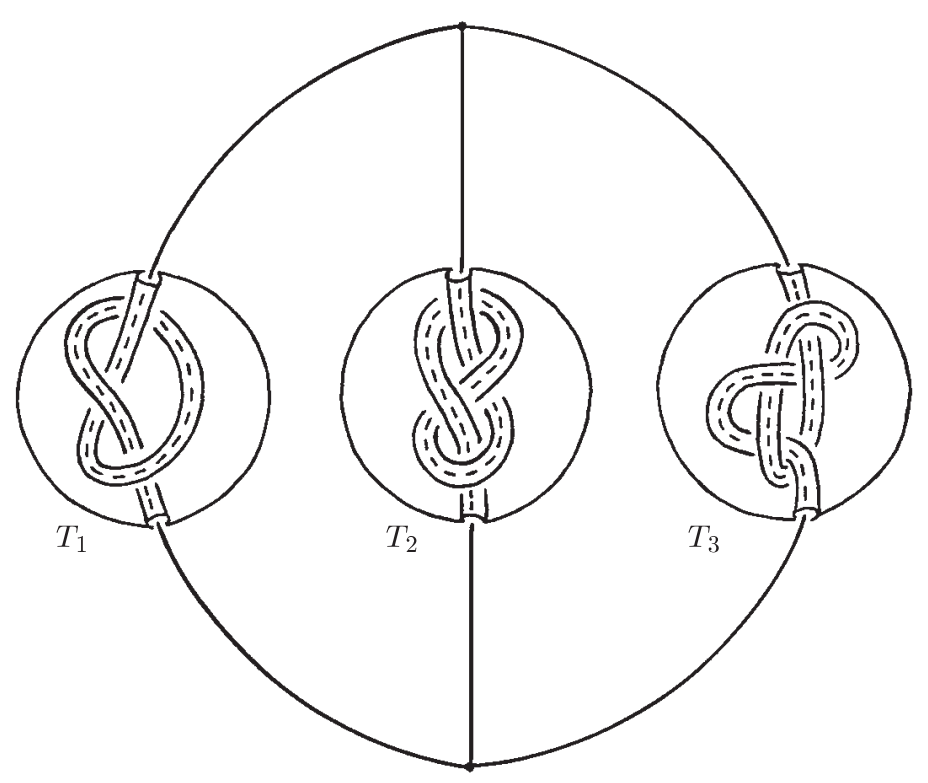

Fig. 1. The decomposing tori for the $\theta$-manifold $M\left(K_{1}, K_{2}, K_{3}\right)$

TheOREM 11. Let $K$ be the trefoil knot in $\mathbb{S}^{3}$. Then the $\theta$-manifolds $M(K, K, K)$ and $M(K, K, \bar{K})$ are not homeomorphic but they possess the same spine. Furthermore, they are the unique compact 3-manifolds with connected boundary having as spine the canonical 2-complex $K_{G}$ associated to the group presentation

$$
G=\left\langle a_{i}, b_{i}, i=1,2,3: a_{i}^{3} b_{i}^{-2}=1, i=1,2,3, a_{1}^{-1} b_{1} a_{2}^{-1} b_{2} a_{3}^{-1} b_{3}=1\right\rangle .
$$

We give only a sketch of the proof (note that one can construct other examples starting from any invertible nonamphicheiral knot). The manifolds of the statement are irreducible and have incompressible boundaries so the fundamental group is not a nontrivial free product. The three annuli, mentioned above, are the characteristic varieties of the manifolds so they must be preserved under homeomorphism, by the Characteristic variety theorem, of $M(K, K, K)$ to $M(K, K, \bar{K})$. This however would mean that the complement of one of the positive trefoil is homeomorphic to the complement of the negative trefoil. However, this is not possible as the trefoil is nonamphicheiral. Finally, invoke the algorithm of Theorem 2 to show that $M(K, K, K)$ and $M(K, K, \bar{K})$ are the unique compact 3 -manifolds having $K_{G}$ as spine.

These results are related to the following question, first settled in [20], concerning regular neighborhoods of homotopically PL embedded two-dimensional polyhedra in 3manifolds. For a discussion in higher dimension we refer to [7].

Problem 7. Let $K$ be a 2 -polyhedron and let $f_{1}, f_{2}: K \rightarrow \stackrel{\circ}{\text { be two (homotopic) }}$ embeddings of $K$ into the interior of a compact 3-manifold $M$. Let $N_{i} \subset M$ be a regular neighborhood of $f_{i}(K)$ in $M, i=1,2$. Under which conditions, are $N_{1}$ and $N_{2}$ homeomorphic? 
In [2] and [20] it was shown that $N_{1}$ and $N_{2}$ are homeomorphic for many classes of special polyhedra (compare also [1]). Indeed, any special polyhedron thickens to at most one 3-manifold. In [2] the following result was proved.

THEOREM 12. Let $M$ be a closed irreducible 3-manifold which is not a fake 3-sphere. If $\partial N_{i} \cong \mathbb{S}^{2}$, then $N_{1}$ is homeomorphic to $N_{2}$.

We can therefore state the following conjecture.

CONJecture. Let $M$ be a homotopy 3-sphere (or 3-cell), $K$ a simply-connected 2polyhedron, $f_{1}, f_{2}: K \rightarrow \stackrel{\circ}{M}$ embeddings and $N_{i}$ a regular neighborhood of $f_{i}(K)$ in $M$. If $\partial N_{i} \cong \mathbb{S}^{2}$, then $N_{1} \cong N_{2}$.

This conjecture is interesting because if it is true, then the 3-dimensional Poincaré conjecture is proved to be equivalent to the following statement:

Every homotopy 3 -cell possesses a spine which embeds in $\mathbb{R}^{3}$.

Indeed, the Poincaré conjecture implies this statement without any additional conjecture. Conversely, let $M$ be a homotopy 3-cell and choose a (tame) 3-cell $C$ in the interior of $M$. By hypothesis, $M$ has a spine $K \subset \stackrel{M}{ }$ which embeds in the interior of $C$ via a

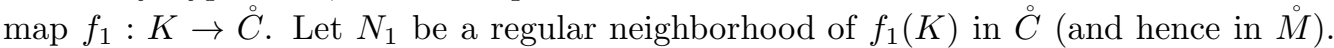
It follows immediately that $N_{1}$ is a genuine 3-cell. Furthermore, $N_{2}:=M$ is a regular neighborhood of $f_{2}(K)=K$ in $M$, where $f_{2}$ is the inclusion map. The above conjecture now implies that the 3 -cell $N_{1}$ is homeomorphic to $M$, as requested.

\section{References}

[1] B. G. Casler, An embedding theorem for connected 3-manifolds with boundary, Proc. Amer. Math. Soc. 16 (1965), 559-566.

[2] A. Cavicchioli, Imbeddings of polyhedra in 3-manifolds, Annali di Mat. Pura ed Appl. 162 (1992), 157-177.

[3] A. Cavicchioli, Neuwirth manifolds and colourings of graphs, Aequationes Math. 44 (1992), 168-187.

[4] A. Cavicchioli and F. Hegenbarth, Knot manifolds with isomorphic spines, Fund. Math. 145 (1994), 79-89.

[5] A. Cavicchioli, F. Hegenbarth and A. C. Kim, A geometric study of Sieradski groups, Algebra Colloq. 5 (1998), to appear.

6] A. Cavicchioli, W. B. R. Lickorish and D. Repovš, On the equivalent spines problem, Boll. Un. Mat. Ital., to appear.

[7] A. Cavicchioli and D. Repovš, Peripheral acyclicity and homology manifolds, Annali di Mat. Pura ed Appl. 172 (1997), 5-24.

[8] A. Cavicchioli and F. Spaggiari, The classification of 3-manifolds with spines related to Fibonacci groups, in "Algebraic Topology-Homotopy and Group Cohomology", Lect. Notes in Math., Springer Verlag 1509 (1992), 50-78.

[9] H. Helling, A. C. Kim and J. L. Mennicke, A geometric study of Fibonacci groups, Preprint Universitat Bielefeld 343 (1990). 
[10] H. M. Hilden, M. T. Lozano and J. M. Montesinos, The arithmeticity of the figure eight knot orbifolds, in "Topology '90", Walter de Gruyter Ed., Berlin - New York (1992), $169-183$.

[11] C. Hog-Angeloni, W. Metzler and A. J. Sieradski, Two-dimensional homotopy and combinatorial group theory, London Math. Soc. Lect. Note Ser. 197, Cambridge Univ. Press, Cambridge, 1993.

[12] J. Howie, Cyclic presentations and $(2,2 k+1)$ torus knots, unpublished.

[13] A. C. Kim, On the Fibonacci group and related topics, Contemporary Math. 184 (1995), 231-235.

[14] D. L. Johnson and W. K. Odoni, Some results on symmetrically presented groups, Proceed. Edinburgh Math. Soc. 37 (1994), 227-237.

[15] A. Mednykh and A. Vesnin, Hyperbolic volumes of Fibonacci manifolds, Siberian Math. J. 36 (1995), 235-245.

[16] J. L. Mennicke, On Fibonacci groups and some other groups, Proceed. of GroupsKorea 1988, Pusan, August 1988, 117-123.

[17] J. Milnor, On the 3-dimensional Brieskorn manifolds $M(p, q, r)$, in "Knots, Groups and 3-Manifolds" (L. P. Neuwirth Ed.), Ann. of Math. Studies 84, Princeton Univ. Press, Princeton, N. J., 1975, 175-225.

[18] W. J. R. Mitchell, J. H. Przytycki and D. Repovš, On spines of knot spaces, Bull. Polish Acad. Sci. 37 (1989), 563-565.

[19] L. Neuwirth, An algorithm for the construction of 3-manifolds from 2-complexes, Proc. Camb. Phil. Soc. 64 (1968), 603-613.

[20] D. Repovš, Regular neighbourhoods of homotopically PL embedded compacta in 3-manifolds, Suppl. Rend. Circ. Mat. Palermo 18 (1988), 415-422.

[21] P. Scott, The Geometries of 3-manifolds, Bull. London Math. Soc. 15 (1983), 401-487.

[22] A. J. Sieradski, Combinatorial squashings, 3-manifolds, and the third homotopy of groups, Invent. Math. 84 (1986), 121-139.

[23] R. Thomas, On a question of Kim concerning certain group presentations, Bull. Korean Math. Soc. 28 (1991), 219-244. 